\title{
Quality of life in patients with brain metastases receiving upfront as compared to salvage stereotactic radiosurgery using the EORTC QLQ-C15-PAL and the EORTC QLQ BN20+2: a pilot study
}

\author{
Kaitlin Koo • Marc Kerba • Liang Zeng • Liying Zhang • \\ Emily Chen $\cdot$ Edward Chow $\cdot$ Arjun Sahgal \\ Received: 28 May 2012 / Accepted: 21 June 2012 / Published online: 26 July 2012 \\ (C) Springer-Verlag 2012
}

\begin{abstract}
Objective The purpose of this study is to investigate quality of life (QOL) outcomes in patients receiving either upfront (no prior whole brain radiation) or salvage (prior whole brain radiotherapy) stereotactic radiosurgery (SRS) for brain metastases using the European Organization for Research and Treatment of Cancer (EORTC) QLQ-C15-PAL and the EORTC QLQ-BN20+2 questionnaires.

Methods Thirty patients who received either upfront or salvage SRS for brain metastases completed the two questionnaires before and 4 weeks following SRS. Scale scores at baseline and at the 1-month follow-up were calculated using the EORTC scoring manual.

Results Fifteen patients were treated with upfront SRS and 15 with salvage SRS. The baseline characteristics of the two groups were balanced with the exception of more patients in the salvage cohort having had received prior systemic therapy (93\% vs. $40 \% ; p=0.0052$, respectively). When
\end{abstract}

K. Koo $\cdot$ L. Zeng $\cdot$ L. Zhang $\cdot$ E. Chen $\cdot$ E. Chow $\cdot$ A. Sahgal Rapid Response Radiotherapy Program, Odette Cancer Centre Sunnybrook Health Sciences Centre,

Toronto, ON, Canada

M. Kerba

Tom Baker Cancer Centre, University of Calgary,

Calgary, AB, Canada

\section{A. Sahgal}

Princess Margaret Hospital, University Health Network, University of Toronto,

Toronto, ON, Canada

\section{A. Sahgal $(\bowtie)$}

Department of Radiation Oncology, Odette Cancer Centre,

Sunnybrook Health Sciences Centre,

2075 Bayview Avenue,

Toronto, ON, Canada M4N 3 M5

e-mail: arjun.sahgal@sunnybrook.ca comparing QOL outcomes at baseline and at 1 month post-SRS, no significant differences in QOL were observed between cohorts based on the EORTC QLQ C15-PAL and the EORTC QLQ-BN20+2 change scales.

Conclusion Patients receiving salvage SRS maintain a similar QOL profile as compared to patients treated upfront with SRS at 1-month posttreatment. Our findings support the use of salvage SRS in patients with brain relapse following prior WBRT.

Keywords Brain metastases - Stereotactic radiosurgery · Palliative radiation - Quality of life - QLQ-BN20 . QLQ-C15-PAL

\section{Introduction}

Metastatic brain tumors are a common complication in patients with advanced cancer occurring in about 20-40\% of all cancer patients $[1,2]$. The most common primary cancers that metastasize to the brain include lung, breast, and skin (melanoma) [3].

For the patient presenting with a limited number of brain metastases, several patient and tumor factors are considered prior to recommending a treatment course. These include the patient's performance status, whether solitary or multiple (up to four vs more than four tumors), the presence of systemic metastases, whether or not the primary tumor is controlled, availability of further systemic therapies and age [3-5]. For good performance status patients with a reasonably long life expectancy, controlled systemic disease, and up to four brain metastases, treatment options include whole brain radiotherapy (WBRT) alone, WBRT plus stereotactic radiosurgery (SRS) boost, or SRS alone. A recent metaanalysis reviewed the impact of these options [6] and found 
no survival advantage to the use of WBRT plus a SRS boost as compared to SRS alone. However, fewer distant relapses and local failures were observed with combined WBRT plus SRS boost. These gains from WBRT do come at a cost of impaired neurocognitive functioning [7], and why SRS alone has emerged as a first-line therapeutic option.

For patients who have been previously treated with WBRT and relapsed intra-cranially, treatment options consist of repeat WBRT, SRS as a salvage focal therapy or supportive care alone. Typically, repeat WBRT is avoided and used in those patients with a poor life expectancy or widely disseminated brain disease. Neurotoxicity may be quite significant in the long-term with repeat WBRT and, furthermore, its efficacy is questionable as the dose of radiation is lower the second time such that we do not necessarily expect long-term local tumor control. Therefore, in patients who are fit and have a limited number of tumors to be treated, salvage SRS is a preferred treatment option.

There are limited data with respect to efficacy [6-8] for salvage SRS and no randomized trials comparing outcomes to repeat WBRT or even supportive care alone. In particular, we lack quality of life (QOL) outcomes for this cohort to help us better understand the impact of salvage SRS on our patient's life. QOL outcomes are highly relevant as QOL is a multidimensional construct comprised of physical, psychological, social well-being, and functioning as perceived by the individual. Some argue that QOL is the most relevant factor in guiding patients in their decision making process.

The aim of this prospective study was to conduct a pilot investigation to determine preliminary QOL outcomes in a cohort of patients treated with salvage SRS and to compare outcomes to patients treated with upfront SRS using the validated European Organization for Research and Treatment of Cancer (EORTC) QLQ-C15-PAL and the EORTC QLQ-BN20+2 questionnaires.

\section{Methods}

\section{Patients}

This study included 30 patients with brain metastases treated with SRS from three Canadian cancer centers from May 2009 to August 2010. All patients had to be eligible for SRS according to the treating radiation oncologist and consent to the study. Fifteen patients had been treated with upfront SRS and 15 patients with salvage SRS (Table 1). Patients completed the EORTC QLQ-C15-PAL and the EORTC QLQ-BN20+2 questionnaires before and at 1 month following SRS treatment. All researches were conducted with approval from each institution's research ethics board. Baseline patient data included: age, Karnofsky Performance Status (KPS), sex, primary cancer site, the presence of co-existing visceral or bone metastases, whether the patient had been treated with SRS upfront or as salvage for relapse following prior WBRT, and each patient was scored according to the Graded Prognostic Assessment (GPA) scale [9]. Patients treated with boost SRS were excluded. Concurrent systemic treatments such as chemotherapy, hormone therapy, and bisphosphonates within 1 week of baseline were also recorded.

\section{Questionnaires}

QOL for patients receiving SRS was assessed using the EORTC QLQ-BN20+2 and the EORTC QLQ-C15-PAL questionnaires (Figs. 1 and 2). Patients completed the questionnaires in person at baseline (date of initial SRS consultation) and by telephone with a research assistant at 1 month following the date of initiation of SRS treatment. The QLQC15-PAL is a general core QOL assessment tool that can be used in conjunction with various cancer subtype specific questionnaires. The QLQ-C15-PAL [10] consists of 15 questions that encompass multi-item functional scales (for example, physical and emotional functioning), multi-item symptom scales (for example, fatigue and pain), singleitem symptom scales (for example, nausea, vomiting, dyspnea, insomnia, appetite loss, and constipation), and a final question evaluating the patients' overall QOL (Fig. 1).

The QLQ-C15-PAL core questionnaire was used in conjunction with the QLQ-BN20+2, which is a brain-specific subtype of the questionnaire. The QLQ-BN20+2 [11] consists of 22 questions that encompass single-item symptom scales (for example, headaches, seizures, drowsiness, hair loss, itchy skin, leg weakness, and bladder control), multiitem scales (for example, future uncertainty, visual disorder, motor dysfunction, and communication deficit), and cognitive functioning (for example, concentration and memory) scales (Fig. 2).

\section{Statistical analysis}

To compare demographic characteristics between patients treated with upfront SRS to those with salvage SRS, the Fisher exact test was applied for categorical variables (i.e., gender, marital status, primary cancer site, etc.), and the Student's $t$ test for continuous variables (i.e., age, KPS, and GPA). Questionnaire scores were tabulated using the EORTC QLQ C30 Scoring Manual in addition to the manuals' addendum entitled: EORTC QLQ-C15-PAL scoring. Scaled scores (range, $0-100 \%$ ) were calculated for the EORTC QLQ-C15-PAL and the EORTC QLQ BN20+2 (cognitive items) questionnaires. For the QLQ-C15-PAL, the higher the functional/global health status QOL scores the better the QOL. However, the higher the symptom score, 
Table 1 Baseline demographics of the upfront SRS cohort compared to the salvage SRS cohort
$P$ value was obtained by Fisher exact test for categorical variables or Student's $t$ test for continuous variables (age, KPS, and GPA). $P$ value $<0.05$ was considered as statistical significance

\begin{tabular}{|c|c|c|c|}
\hline & Upfront SRS cohort & Salvage SRS cohort & $P$ value \\
\hline Age (years) & & & 0.48 \\
\hline Number & 15 & 15 & \\
\hline Mean \pm SD & $60.3 \pm 9.1$ & $57.9 \pm 9.8$ & \\
\hline Interquartiles & $54.0-71.0$ & $49.0-64.0$ & \\
\hline Median (range) & $59(48-77)$ & $57(44-79)$ & \\
\hline KPS & & & 0.89 \\
\hline Number & 14 & 14 & \\
\hline Mean \pm SD & $78.6 \pm 15.1$ & $79.3 \pm 12.1$ & \\
\hline Interquartiles & $70-90$ & $70-90$ & \\
\hline Median (range) & $80(50-100)$ & $85(60-90)$ & \\
\hline Gender & & & 0.14 \\
\hline Female & $6(40.0 \%)$ & $11(73.3 \%)$ & \\
\hline Male & $9(60.0 \%)$ & $4(26.7 \%)$ & \\
\hline GPA & & & 0.51 \\
\hline Number & 15 & 15 & \\
\hline Mean \pm SD & $1.9 \pm 0.8$ & $2.1 \pm 0.9$ & \\
\hline Interquartiles & $1.5-2.5$ & $1.5-3.0$ & \\
\hline Median (range) & $2.0(0.5-3)$ & $2.5(0.5-3)$ & \\
\hline Marital status & & & 0.28 \\
\hline Married & $10(66.7 \%)$ & $11(73.3 \%)$ & \\
\hline Single & $1(6.6 \%)$ & $3(20.0 \%)$ & \\
\hline Other & $4(26.7 \%)$ & $1(6.7 \%)$ & \\
\hline Primary cancer site & & & 0.37 \\
\hline Lung & $8(53.3 \%)$ & $11(73.3 \%)$ & \\
\hline Breast & $1(6.7 \%)$ & $2(13.3 \%)$ & \\
\hline Colon & $1(6.7 \%)$ & $0(0.0 \%)$ & \\
\hline Melanoma & $2(13.3 \%)$ & $1(6.7 \%)$ & \\
\hline Renal cell/kidney & $3(20 \%)$ & $0(0.0 \%)$ & \\
\hline Ovarian & $0(0.0 \%)$ & $1(6.7 \%)$ & \\
\hline Number of brain metastases & & & 0.40 \\
\hline 1 & $8(53.3 \%)$ & $4(26.7 \%)$ & \\
\hline 2 to 3 & $5(33.3 \%)$ & $8(53.3 \%)$ & \\
\hline$>3$ & $2(13.3 \%)$ & $3(20.0 \%)$ & \\
\hline Previous systemic therapy & & & 0.0052 \\
\hline Yes & $6(40.0 \%)$ & $14(93.3 \%)$ & \\
\hline No & $9(60.0 \%)$ & $1(6.7 \%)$ & \\
\hline
\end{tabular}

the lower the QOL. For the BN20+2, higher scores indicate worse QOL.

Time periods were split according to prior to SRS (baseline), and 1 month after SRS (follow-up). Descriptive statistics detailed changes (follow-up-baseline) between these two time periods. To compare C15-PAL change scores, or $\mathrm{BN} 20+2$ change scores, between patients with two different treatments, the Student's $t$ test was used under the assumption of unequal variance. To normalize the distribution, a natural log-transformation was applied for each change score. To adjust for multiple comparisons, a Bonferroni adjusted $p$ value of $<0.004(0.05 / 13$ scale scores $)$ was considered as statistically significant. All analyses were conducted using the Statistical Analysis Software (SAS version 9.2 for Windows).

\section{Results}

Baseline demographics for patients treated with upfront SRS

For the 15 patients receiving upfront SRS, six were female and nine male, the median age was 59 years (range, 4877 years), the median KPS was 80 (range, 50-100), and the 
Fig. 1 EORTC QLQ-C15-PAL questionnaire

\section{EORTC QLQ - C15 - PAL (version 1)}

We are interested in some things about you and your health. Please answer all of the questions yourself by circling the number that best applies to you. There are no "right" or "wrong" answers. The information that you provide will remain strictly confidential.

\section{Not at A Quite Very \\ All Little a Bit Much}

$\begin{array}{lllll}\text { 1. Do you have any trouble taking a short walk outside the house? } & 1 & 2 & 3 & 4 \\ \text { 2. Do you need to stay in bed or a chair during the day? } & 1 & 2 & 3 & 4 \\ \text { 3. Do you need help with eating, dressing, washing yourself } & 1 & 2 & 3 & 4\end{array}$

\section{During the Past Week:}

4. Were you short of breath?

5. Have you had pain?

6. Have you had trouble sleeping?

7. Have you felt weak?

8. Have you lacked appetite?

9. Have you felt nauseated?

10. Have you been constipated?

11. Were you tired?

12. Did pain interfere with your daily activities?

13. Did you feel tense?

14. Did you feel depressed?

\section{Not at A Quite Very \\ All Little a Bit Much}

$\begin{array}{llll}1 & 2 & 3 & 4 \\ 1 & 2 & 3 & 4 \\ 1 & 2 & 3 & 4 \\ 1 & 2 & 3 & 4 \\ 1 & 2 & 3 & 4 \\ 1 & 2 & 3 & 4 \\ 1 & 2 & 3 & 4 \\ 1 & 2 & 3 & 4 \\ 1 & 2 & 3 & 4 \\ 1 & 2 & 3 & 4 \\ 1 & 2 & 3 & 4\end{array}$

For the following question please circle the number between 1 and 7 that best applies to you. 15. How would you rate your overall quality of life during the past week?

$\begin{array}{cccccccc}1 & 2 & 3 & 4 & 5 & 6 & 7 \\ \text { Very poor } & & & & & & & \\ \text { Excellent }\end{array}$

median GPA score was 2 (range, 0.5-3). The most common sites of primary cancer included lung $(53.3 \%)$, renal cell/ kidney $(20.0 \%)$, melanoma (13.3\%), colon $(6.7 \%)$, and breast $(6.7 \%) ; 53.3 \%, 33.3 \%$, and $13.3 \%$ of the patients had $1,2-3$, or $>3$ brain metastases treated with SRS (Table 1).

Baseline demographics for patients treated with SRS as salvage

For the 15 patients receiving SRS as salvage, 11 were female and four were male, the median age was 57 years (range, 44-79 years), the median KPS was 85 (range,
60-90), and the median GPA score was 2.5 (range, 0.5$3)$. The most common sites of primary cancer included lung $(73.3 \%)$, breast $(13.3 \%)$, melanoma $(6.7 \%)$, and ovarian $(6.7 \%) ; 26.7 \%, 35.3 \%$, and $20 \%$ of the patients had $1,2-3$, or $>3$ brain metastases treated with SRS (Table 1).

Comparison of baseline characteristics between the upfront SRS and SRS salvage cohort

Patients treated with salvage SRS were more likely to have received systemic therapy as compared to those treated with upfront SRS (93\% vs. $40 \% ; p=0.0052$, respectively). 
Fig. 2 EORTC QLQ-BN20 + 2 questionnaire

\section{EORTC QLQ-BN20 + 2 Cognitive Items}

Patients sometimes report that they have the following symptoms. Please indicate the extent to which you have experienced these symptoms or problems during the past week.

\begin{tabular}{|c|c|c|c|c|}
\hline During the past week: & $\begin{array}{l}\text { Not at } \\
\text { all }\end{array}$ & A little & $\begin{array}{l}\text { Quite a } \\
\text { bit }\end{array}$ & $\begin{array}{l}\text { Very } \\
\text { much }\end{array}$ \\
\hline 1. Did you feel uncertain about the future? & 1 & 2 & 3 & 4 \\
\hline 2. Did you feel you had setbacks in your condition? & 1 & 2 & 3 & 4 \\
\hline 3. Were you concemed about disruption of family life? & 1 & 2 & 3 & 4 \\
\hline 4. Did you have headaches? & 1 & 2 & 3 & 4 \\
\hline 5. Did your outlook on the future worsen? & 1 & 2 & 3 & 4 \\
\hline 6. Did you have double vision? & 1 & 2 & 3 & 4 \\
\hline 7. Was your vision blurred? & 1 & 2 & 3 & 4 \\
\hline 8. Did you have difficulty reading because of your vision? & 1 & 2 & 3 & 4 \\
\hline 9. Did you have seizures? & 1 & 2 & 3 & 4 \\
\hline 10. Did you have weakness on one side of your body? & 1 & 2 & 3 & 4 \\
\hline $\begin{array}{l}\text { 11. Did you have trouble finding the right words to express } \\
\text { yourself }\end{array}$ & 1 & 2 & 3 & 4 \\
\hline 12. Did you have difficulty speaking? & 1 & 2 & 3 & 4 \\
\hline 13. Did you have trouble communicating your thoughts? & 1 & 2 & 3 & 4 \\
\hline 14. Did you feel drowsy during the daytime? & 1 & 2 & 3 & 4 \\
\hline 15. Did you have trouble with your coordination? & 1 & 2 & 3 & 4 \\
\hline 16. Did hair loss bother you? & 1 & 2 & 3 & 4 \\
\hline 17. Did itching of your skin bother you? & 1 & 2 & 3 & 4 \\
\hline 18. Did you have weakness of both legs? & 1 & 2 & 3 & 4 \\
\hline 19. Did you feel unsteady on your feet? & 1 & 2 & 3 & 4 \\
\hline 20. Did you have trouble controlling your bladder? & 1 & 2 & 3 & 4 \\
\hline \multicolumn{5}{|l|}{ 21. Have you had difficultly in concentrating on things, } \\
\hline like reading a newspaper or watching television? & 1 & 2 & 3 & 4 \\
\hline 22. Have you had difficulty remembering things? & 1 & 2 & 3 & 4 \\
\hline
\end{tabular}

Otherwise no significant differences between the two treatments cohorts were observed (Table 1).

Comparing QOL outcomes based on changes from baseline to the 1 month follow-up in the upfront SRS vs. salvage SRS cohort

Table 2 summarizes the EORTC QLQ-C15-PAL scaled scores from baseline to 1-month follow-up in patients treated with upfront SRS and salvage SRS. When comparing QOL outcomes using the Bonferroni adjusted $p$ value of $<0.004$ as statistically significant, no significant differences were observed. Table 3 summarizes the EORTC QLQBN20+2 scaled scores from baseline to 1 month follow-up in patients treated with upfront SRS and salvage SRS. When comparing QOL outcomes using the Bonferroni adjusted $p$ value of $<0.004$ as statistically significant, at baseline and at 1 month post-SRS, no significant differences in QOL were observed.

\section{Discussion}

The current literature assessing the impact of SRS for brain metastases tends to emphasize endpoints such as survival, and time to local and distant intracranial recurrence $[6,12$, 13]. Although these endpoints are critical considerations to justify the resource intensive and costly therapy of SRS [14], the importance of QOL has been less emphasized and should be considered equally critical in the treatment 
Table 2 Comparison of changes in EORTC QLQ-C15-PAL scaled scores from baseline to 1 month follow-up in patients treated with upfront SRS and salvage SRS

\begin{tabular}{|c|c|c|c|c|c|c|c|}
\hline \multirow[t]{2}{*}{ Item } & \multicolumn{3}{|c|}{ Upfront SRS cohort $(n=15)$} & \multicolumn{3}{|c|}{ Salvage SRS cohort $(n=15)$} & \multirow{2}{*}{$\begin{array}{l}\text { Comparison } \\
p \text { value }^{\mathrm{a}}\end{array}$} \\
\hline & Baseline (mean) & $\begin{array}{l}1 \text { month follow-up } \\
\text { (mean) }\end{array}$ & Difference & Baseline (mean) & $\begin{array}{l}1 \text { month follow-up } \\
\text { (mean) }\end{array}$ & Difference & \\
\hline Pain Scale & 23.1 & 28.2 & 5.1 & 23.6 & 16.7 & -6.9 & 0.41 \\
\hline Dyspnea & 11.1 & 17.8 & 6.7 & 15.6 & 11.1 & -4.4 & 0.28 \\
\hline Insomnia & 31.1 & 20.0 & -11.1 & 24.4 & 22.2 & -2.2 & 0.34 \\
\hline Appetite loss & 9.5 & 22.2 & 11.9 & 20.0 & 15.6 & -4.4 & 0.15 \\
\hline Constipation & 17.8 & 4.4 & -13.3 & 11.1 & 11.1 & 0.0 & 0.27 \\
\hline QOL scale & 70.0 & 65.6 & -4.4 & 65.6 & 63.3 & -2.2 & 0.60 \\
\hline Physical Functioning & 72.9 & 73.3 & 0.4 & 70.2 & 70.7 & 0.4 & 0.99 \\
\hline Fatigue & 38.5 & 45.9 & 12.0 & 35.6 & 39.3 & 3.7 & 0.22 \\
\hline Nausea and vomiting & 5.6 & 14.4 & 8.9 & 14.4 & 2.2 & -12.2 & 0.18 \\
\hline Emotional Functioning & 84.4 & 80.0 & -4.4 & 82.2 & 88.1 & 6.0 & 0.23 \\
\hline
\end{tabular}

${ }^{a} P$ value was obtained by applying the Student's $t$ test. A Bonferroni adjusted $p$ value of $<0.004$ was considered as statistically significant. Natural log-transformation was applied for each change score to normalize distribution

decision-making process. In particular, in the salvage SRS patient, there are no randomized trials to justify SRS; however, it is acknowledged that for selected patients, long-term efficacy and survival has been observed $[8,15,16]$. The issue lies in whether we are improving, or at least even maintaining, QOL following this intense treatment. To our knowledge, this study is the first to report defined QOL outcomes using a validated QOL instrument in a population of patients treated with salvage SRS, and compare outcomes to those treated with upfront SRS.
The present pilot study was designed as a feasibility study with respect to collecting QOL outcomes in the salvage SRS population and to gain preliminary outcomes to base a larger study upon. This is why our data collection was limited to the 1-month posttreatment endpoint as a future planned study on a large scale will address long-term follow-up.

We first observed that the two cohorts were well balanced (Table 1). However, patients in the salvage SRS cohort were more likely to have been treated with previous systemic

Table 3 Comparison of changes in EORTC QLQ BN20 + 2 scaled scores from baseline to 1 month follow-up in patients treated with upfront SRS and salvage SRS

\begin{tabular}{|c|c|c|c|c|c|c|c|}
\hline \multirow[t]{2}{*}{ Item } & \multicolumn{3}{|c|}{ Upfront SRS cohort $(n=15)$} & \multicolumn{3}{|c|}{ Salvage SRS cohort $(n=15)$} & \multirow{2}{*}{$\begin{array}{l}\text { Comparison } \\
p \text { value }^{\mathrm{a}}\end{array}$} \\
\hline & Baseline (mean) & $\begin{array}{l}1 \text { month follow-up } \\
\text { (mean) }\end{array}$ & Difference & Baseline (mean) & $\begin{array}{l}1 \text { month follow-up } \\
\text { (mean) }\end{array}$ & Difference & \\
\hline Future uncertainty & 23.3 & 26.7 & 3.3 & 31.1 & 7.2 & -23.9 & 0.035 \\
\hline Visual disorder & 11.9 & 7.1 & -4.8 & 11.1 & 4.0 & -4.8 & 0.92 \\
\hline Motor dysfunction & 17.8 & 14.1 & -3.7 & 14.8 & 13.3 & -1.5 & 0.53 \\
\hline Communication deficit & 12.8 & 15.4 & 2.6 & 4.8 & 2.4 & -2.4 & 0.26 \\
\hline Headaches & 17.8 & 20.0 & 2.2 & 24.4 & 13.3 & -11.1 & 0.85 \\
\hline Seizures & 2.2 & 0.0 & -2.2 & 0.0 & 0.0 & 0.0 & 0.33 \\
\hline Drowsiness & 31.1 & 44.4 & 13.3 & 21.4 & 28.9 & 4.8 & 0.35 \\
\hline Itchy Skin & 7.7 & 17.9 & 10.2 & 23.8 & 33.3 & 9.5 & 0.39 \\
\hline Hair loss & 0.0 & 8.3 & 8.3 & 20.0 & 11.9 & -6.7 & 0.34 \\
\hline Weakness of legs & 13.3 & 17.8 & 4.4 & 11.1 & 7.1 & -4.8 & 0.48 \\
\hline Bladder control & 0.0 & 2.2 & 2.2 & 6.7 & 7.1 & 2.4 & 0.88 \\
\hline Concentration & 5.9 & 1.5 & -4.4 & 7.4 & 3.0 & -4.4 & 0.93 \\
\hline Memory & 15.4 & 23.1 & 7.7 & 19.0 & 19.0 & 0.0 & 0.77 \\
\hline
\end{tabular}

${ }^{\text {a }} P$ value was obtained by applying the Student's $t$ test. A Bonferroni adjusted $p$ value of $<0.004$ was considered as statistically significant. Natural log-transformation was applied for each change score to normalize distribution 
therapies. This result was not unexpected. From our clinical experience, these patients are further along the course of their disease, and following initial treatment of WBRT are often treated with multiple lines of systemic therapy prior to developing intracranial relapse. The overall similarity of the two cohorts highlights that we are in fact selecting our patients for salvage SRS well. Therefore, it is important to note that our findings are specific to a well-selected cohort of patients with intracranial relapse, and may not be generalizable to all patients who suffer relapse post-WBRT.

We observed in both the upfront and salvage SRS cohort a mixture of improvements and declines in QOL outcomes (Tables 2 and 3) at 1 month post-SRS. In the salvage SRS cohort, we also observed that constipation, seizures, and memory scales did not demonstrate any changes at 1 month post SRS. The common factors that improved 1 month postSRS in both the upfront and salvage SRS cohorts included insomnia, motor dysfunction, communication deficiency, and concentration. The common factors that deteriorated post-SRS in both the upfront and salvage SRS cohorts included global QOL, fatigue, emotional functioning drowsiness, itchy skin, and bladder control. These data are helpful to gain insight into the positive and negative impact of SRS on patient QOL. Statistical analysis comparing QOL outcomes between the two patient cohorts indicated no significant differences based on either the EORTC QLQ-C15PAL or EORTC QLQ-BN20+2 QOL assessment tools. Therefore, in a well-selected cohort of patients with intracranial relapse following prior WBRT, a QOL profile similar to those patients treated with upfront SRS was observed 1 month posttreatment.

\section{Conclusion}

The current study indicates that patients treated with salvage SRS have a comparable QOL profile to those patients treated with upfront SRS 1 month posttreatment. Therefore, a patient who is fit enough for salvage SRS should not be discriminated upon by the advanced state of their disease and offered this aggressive focal therapy. This study also indicates that collecting QOL outcomes in this population is feasible, and larger studies with long-term follow-up are required in order to appreciate the full extent of the impact of salvage SRS on QOL before final conclusions can be rendered.

Acknowledgements We thank the generous support of Bratty Family Fund, Michael and Karyn Goldstein Cancer Research Fund, Joseph and Silvana Melara Cancer Research Fund, and Ofelia Cancer Research Fund.

Conflict of interest The authors have no conflict of interest to disclose.

\section{References}

1. Andrews DW, Scott CB, Sperduto PW, Flanders AE, Gaspar LE, Schell MC, Werner-Wasik M, Demas W, Ryu J, Bahary JP, Souhami L, Rotman M, Mehta MP, Curran WJ (2004) Whole brain radiation therapy with or without stereotactic radiosurgery boost for patients with one to three brain metastases: phase III results of the RTOG 9508 randomised trial. Lancet 363(9422):1665-1672

2. Muller-Riemenschneider F, Bockelbrink A, Ernst I, Schwarzbach C, Vauth C, von der Schulenburg JM, Willich SN (2009) Stereotactic radiosurgery for the treatment of brain metastases. Radiother Oncol 91(1):67-74

3. Mehta MP, Rozental JM, Levin AB, Mackie TR, Kubsad SS, Gehring MA, Kinsella J (1992) Defining the role of radiosurgery in the management of brain metastases. Int J Radiat Oncol Biol Phys 24(4):619-625

4. Gaspar L, Scott C, Rotman M, Asbell S, Phillips T, Wasserman T, McKenna WG, Byhardt R (1997) Recursive partitioning analysis (RPA) of prognostic factors in three Radiation Therapy Oncology Group (RTOG) brain metastases trials. Int J Radiat Oncol Biol Phys 37:745-751

5. Sperduto PW, Chao ST, Sneed PK, Luo X, Suh J, Roberge D, Bhatt A, Jensen AW, Brown PD, Shih H, Kirkpatrick J, Schwer A, Gaspar LE, Fiveash JB, Chiang V, Knisely J, Sperduto CM, Mehta M (2010) Diagnosis-specific prognostic factors, indexes, and treatment outcomes for patients with newly diagnosed brain metastases: a multi-institutional analysis of 4,259 patients. Int J Radiat Oncol Biol Phys 77:655-661

6. Tsao M, Xu W, Sahgal A (2011) A meta-analysis evaluating stereotactic radiosurgery, whole-brain radiotherapy, or both for patients presenting with a limited number of brain metastases. Cancer 118(9):2486-2493

7. Chang EL, Wefel JS, Hess KR, Allen PK, Lang FF, Kornguth DG, Arbuckle RB, Swint JM, Shiu AS, Maor MH, Meyers CA (2009) Neurocognition in patients with brain metastases treated with radiosurgery or radiosurgery plus whole-brain irradiation: a randomised controlled trial. Lancet Oncol 10:1037-1044

8. Follwell MJ, Khu KJ, Cheng L, Xu W, Mikulis DJ, Millar BA, Tsao MN, Laperriere NJ, Bernstein M, Sahgal A (2012) Volume specific response criteria for brain metastases following salvage stereotactic radiosurgery and associated predictors of response. Acta Oncol (Stockholm, Sweden) 51(5):629-635

9. Sperduto PW, Berkey B, Gaspar LE, Mehta M, Curran W (2008) A new prognostic index and comparison to three other indices for patients with brain metastases: an analysis of 1,960 patients in the RTOG database. Int J Radiat Oncol Biol Phys 70:510-514

10. Groenvold M, Petersen MA, Aaronson NK, Arraras JI, Blazeby JM, Bottomley A, Fayers PM, de Graeff A, Hammerlid E, Kaasa S, Sprangers MA, Bjorner JB, EORTC Quality of Life Group (2006) The development of the EORTC QLQ-C15-PAL: a shortened questionnaire for cancer patients in palliative care. Eur J Cancer 42:55-64

11. Caissie A, Nguyen J, Chen E, Zhang L, Sahgal A, Clemons M, Kerba M, Arnalot PF, Danjoux C, Tsao M, Barnes E, Holden L, Danielson B, Chow E (2011) Quality of life in patients with brain metastases using the EORTC QLQ-BN20+2 and QLQ-C15-PAL. Int J Radiat Oncol Biol Phys 83(4):1238-1245

12. Tsao MN, Lloyd N, Wong RK, Chow E, Rakovitch E, Laperriere $\mathrm{N}, \mathrm{Xu} \mathrm{W}$, Sahgal A (2012) Whole brain radiotherapy for the treatment of newly diagnosed multiple brain metastases. Cochrane Database Syst Rev 4: CD003869

13. Kocher M, Soffietti R, Abacioglu U, Villa S, Fauchon F, Baumert BG, Fariselli L, Tzuk-Shina T, Kortmann RD, Carrie C, Ben Hassel M, Kouri M, Valeinis E, van den Berge D, Collette S, Collette L, Mueller RP (2010) Adjuvant whole-brain radiotherapy 
versus observation after radiosurgery or surgical resection of one to three cerebral metastases: results of the EORTC 22952-26001 study. J Clin Oncol 29:134-141

14. Sahgal A, Ma L, Chang E, Shiu A, Larson DA, Laperriere N, Yin FF, Tsao M, Menard C, Basran PS, Letourneau D, Heydarian M, Beachey D, Shukla V, Cusimano M, Hodaie M, Zadeh G, Bernstein M, Schwartz M (2009) Advances in technology for intracranial stereotactic radiosurgery. Technol Cancer Res Treat 8:271-280
15. Chao ST, Barnett GH, Vogelbaum MA, Angelov L, Weil RJ, Neyman G, Reuther AM, Suh JH (2008) Salvage stereotactic radiosurgery effectively treats recurrences from whole-brain radiation therapy. Cancer 113:2198-2204

16. Maranzano E, Trippa F, Casale M, Costantini S, Anselmo P, Carletti S, Principi M, Caserta C, Loreti F, Giorgi C (2011) Reirradiation of brain metastases with radiosurgery. Radiother Oncol 102(2):192-197 